\title{
Comparison of measurements and predictions of sound propagation in a valley-slope configuration in an inhomogeneous atmosphere
}

\author{
T. Van Renterghem ${ }^{\text {a) }}$ and D. Botteldooren \\ Ghent University, Department of Information Technology, Sint-Pietersnieuwstraat 41, \\ B-9000 Gent, Belgium \\ P. Lercher \\ Medical University of Innsbruck, Section of Social Medicine, Innsbruck, Austria
}

(Received 7 September 2006; revised 27 February 2007; accepted 27 February 2007)

\begin{abstract}
Mountainous areas form a very specific context for sound propagation: There is a particular ground effect and meteorological conditions are often extreme. In this paper, detailed sound propagation calculations are compared to noise measurements accompanied by meteorological observations. The sound source considered is road traffic along the center axis of a valley. Noise levels were measured in two cross sections, at three locations each: one on the valley floor and two on the slopes, up to $166 \mathrm{~m}$ above the source. For the numerical calculations, the rotated Green's function parabolic equation method is used, taking into account the undulation of the terrain and an inhomogeneous atmosphere. Typical parameters of this method were optimized for computational efficiency. Predictions agree with measurements to within $3 \mathrm{dBA}$ up to propagation distances of $1 \mathrm{~km}$, in windless conditions. The calculations further show that the terrain profile is responsible for an increase in sound pressure level at distant, elevated points up to $30 \mathrm{dBA}$ compared to a flat ground situation. Complex temperature profiles account for level changes between $-3 \mathrm{dBA}$ and $+10 \mathrm{dBA}$ relative to a homogeneous atmosphere. This study shows that accurate sound level prediction in a valley-slope configuration requires detailed numerical calculations. (c) 2007 Acoustical Society of America. [DOI: 10.1121/1.2717765]
\end{abstract}

PACS number(s): 43.28.Js, 43.28.Fp, 43.28.En [VEO]

Pages: 2522-2533

\section{INTRODUCTION}

When comparing sound propagation in valley-slope configurations with sound propagation over flat terrain, two main differences are observed.

First, the undulation of the terrain influences sound propagation strongly. At some locations, the receiver is shielded from the source by the terrain. In that case, there is no direct sound path, and sound reaches the receiver only by diffraction and refraction over hills. In other situations, receivers are located on slopes high above the valley floor and have direct visibility of the source. They are exposed to significantly higher sound pressure levels than receivers at the same distance at the level of the valley floor would have. The main reason for this is the reduced ground attenuation. In addition, in concave valleys, multiple reflected sound may converge at the up-slope receiver. ${ }^{1}$ In very narrow valleys, sound reflected on the opposite slopes may also contribute significantly to the overall level. ${ }^{1}$

Second, typical meteorological conditions are found in mountainous regions. There is often a large variability of the meteorological parameters in space and time. Besides the influence that mountains exert on the large-scale wind flow (e.g., channeling along the valley axis and the presence of recirculation zones behind orographic obstacles), some typi-

\footnotetext{
a) Author to whom correspondence should be addressed. Electronic mail: timothy.van.renterghem@intec.ugent.be
}

cal, thermally driven air currents occur in valley-slope configurations (so-called slope winds). Wind parameters were not measured in sufficient detail during the measurement campaign in the underlying study, and will therefore not be considered in this paper.

Temperature effects can be more prominent in valleys compared to flat terrain. The transition from the (stable) nightly temperature inversion situation to an unstable atmosphere during daytime can happen very quickly, once the sun rays reach the valley floor. The valley orientation plays an important role in this respect and might cause a delay of several hours with regard to the moment of temperature inversion breakup. $^{2}$ The width-height ratio of a valley influences both the depth and lifetime of the temperature inversion layer. ${ }^{3}$

In Ref. 4, a meteorological meso-scale model was used to simulate temperature profiles and the development of slope-wind systems in a narrow, two-dimensional valley, in the absence of large-scale winds. This information was then used by a numerical sound particle model. This study yielded large variations of sound levels during the course of a day because of the state of the atmosphere. No measurement campaign was set up to check their findings.

In this paper, simultaneous noise measurements and meteorological measurements were performed in order to check sound propagation calculations. The situation of interest is upslope sound propagation, orthogonal to the valley axis. This corresponds to a typical situation in valley-slope con- 


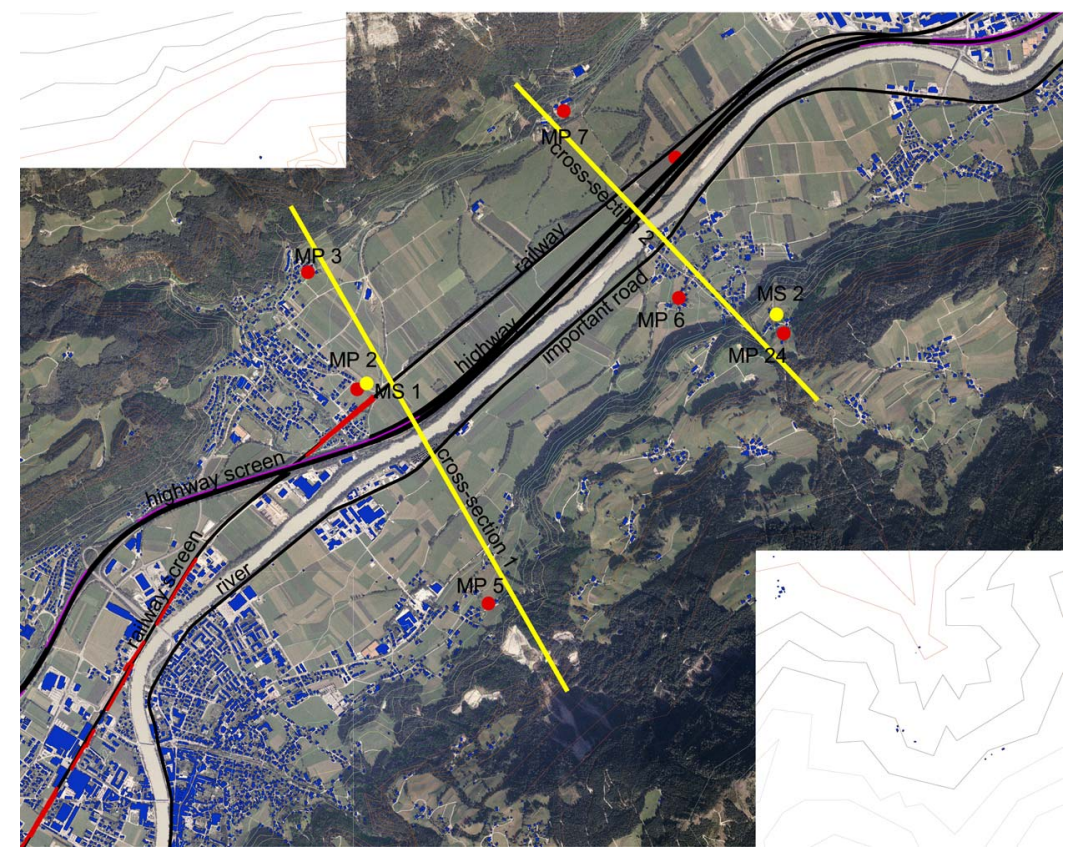

FIG. 1. (Color online) Orthophoto of the valley under consideration. The following items are indicated: the microphone positions (MP), the locations of the meteo stations (MS), the highway, the main road, the railway, the river, the locations of highway screens and railway screens, and the buildings. Height contours are shown as well.

figurations, where a highway or railway follows the valley axis and where dwellings are present on the slopes. A detailed comparison between noise measurements and (wavebased) sound propagation calculations in such situations has not been reported previously. Focus is on the effect of an inhomogeneous atmosphere. Large-scale wind systems will influence sound propagation to a lesser extent in thistypical-configuration since wind direction is usually aligned with the valley axis. Slope winds are directed orthogonal to the valley axis, but are not considered here.

This paper is organized as follows. In Sec. II, the region where the study was performed is described. In Sec. III, the acoustical and meteorological measurement setup is described. It is also indicated how the data was preprocessed. Details on the numerical model and the calculation methodology can be found in Sec. IV and Appendix. A comparison between measurements and predictions is made in Sec. V. In Sec. VI, conclusions are drawn.

\section{SITE DESCRIPTION}

The Unterinntal region is located in the Alps, in the western part of Austria. The measurements were performed in a 2-km-wide valley in the district Schwaz. An orthophoto of the area under consideration is shown in Fig. 1. The valley floor is located at an elevation between 530 and $540 \mathrm{~m}$. The valley is oriented in general from North-East to South-West. The mountains surrounding the valley have ridges of over $2000 \mathrm{~m}$ in height. In the center of the valley, there is a highway (Inntal Autobahn, A12), an important main road (Tiroler Bundesstrasse, B171), and a railway close to the highway.

In this paper, focus is on sound propagation from road sources to a number of locations on the slopes of the valley, at lower elevation. The difference in elevation between the road and the microphone positions is at most $166 \mathrm{~m}$. The presence of dwellings and thus possibly noise annoyed people at higher altitude on the slope is limited.
Two cross sections were selected and in each of them three microphones were placed. The microphone positions in each cross section lie more or less on a straight line, orthogonal to the roads. Near cross section 1, the highway (A12) is the dominant noise source. Near cross section 2 a busy road (B171) dominates the noise climate. An overview of the orthogonal distances to the relevant roads, the ground elevation at the microphone locations, as well as the elevation of the roads near each microphone can be found in Table I. All microphones, with the exception of microphone 6 , are placed $2 \mathrm{~m}$ above the ground. Microphone 6 is placed on the roof of a small building for practical reasons. A portable meteo station is used to gather on-site meteorological data.

Figure 1 shows a map of the area clearly indicating the roads, railway, noise barriers along highway and railway, houses and other buildings, and the measurement locations. Sound measurement stations are identified by the microphone numbers used in Table I.

\section{MEASUREMENTS AND PROCESSING}

The measurement campaign lasted from November 2005 till January 2006. Simultaneous noise measurements and meteorological observations were performed.

TABLE I. Overview of microphone positions during the measurement campaign.

\begin{tabular}{ccccc}
\hline \hline Clevation of & \\
$\begin{array}{c}\text { Orthogonal } \\
\text { distance } \\
\text { towards } \\
\text { section } \\
\text { ID }\end{array}$ & $\begin{array}{c}\text { Microphone } \\
\text { ID }\end{array}$ & $\begin{array}{c}\text { dominant road } \\
\text { noise source } \\
(\mathrm{m})\end{array}$ & $\begin{array}{c}\text { Ele roads }(\mathrm{m}) \\
\text { near } \\
\text { microphone }\end{array}$ & $\begin{array}{c}\text { Elevation at } \\
\text { receiver (m) }\end{array}$ \\
\hline 1 & 2 & 331 & $530-541$ & 540 \\
1 & 3 & 1188 & $530-560$ & 583 \\
1 & 5 & 1216 & $530-563$ & 579 \\
2 & 6 & 542 & $530-540$ & 541 \\
2 & 7 & 796 & $530-540$ & 542 \\
2 & 24 & 1153 & $530-541$ & 696 \\
\hline \hline
\end{tabular}




\begin{tabular}{|c|c|c|c|c|c|c|}
\hline $\begin{array}{l}\text { Cross- } \\
\text { section } \\
\text { ID }\end{array}$ & $\begin{array}{c}\text { Cluster ID } \\
\text { (temperature } \\
\text { profile ID) }\end{array}$ & $\begin{array}{l}\text { Number } \\
\text { of } \\
\text { locations }\end{array}$ & $\begin{array}{l}\text { Number of } \\
\text { retained } \\
\text { measurements } \\
\text { in cluster }\end{array}$ & $\begin{array}{l}\text { Additional } \\
\text { meteo } \\
\text { information } \\
\text { in cross } \\
\text { section }\end{array}$ & $\begin{array}{c}\text { Measurement } \\
\text { period: } \\
\text { day }(\mathrm{s})\end{array}$ & $\begin{array}{c}\text { Measurement } \\
\text { period: } \\
\text { hours }\end{array}$ \\
\hline 1 & 1 & 3 & 53 & Yes (MS 1) & $20 / 11 / 05$ & $\begin{array}{l}16.00- \\
19.00 \mathrm{~h}\end{array}$ \\
\hline 2 & 2 & 3 & 148 & $\begin{array}{c}\text { No (MS } 1 \text { is } \\
\text { used) }\end{array}$ & $\begin{array}{c}26 / 11 / 05- \\
01 / 12 / 05\end{array}$ & $9.00-16.00 \mathrm{~h}$ \\
\hline 2 & 3 & 3 & 67 & $\begin{array}{c}\text { No (MS } 1 \text { is } \\
\text { used) }\end{array}$ & 27/11/05 & $\begin{array}{l}11.00- \\
13.00 \mathrm{~h}\end{array}$ \\
\hline 2 & 4 & 2 & 59 & Yes (MS 2) & $\begin{array}{l}20 / 12 / 05- \\
21 / 12 / 05\end{array}$ & $\begin{array}{l}23.00- \\
08.00 \mathrm{~h}\end{array}$ \\
\hline
\end{tabular}

\section{A. Meteo measurements and processing}

A Vaisala MAWS201 Automatic Weather Station was used to gather basic meteorological information in each cross section. The data consist of air temperature, relative humidity, atmospheric pressure, wind speed, and wind direction, all measured at a single height. Every minute, this data was logged. The wind speed was measured with an anemometer at $2 \mathrm{~m}$ above the ground. This information is insufficient to estimate wind speed profiles. It nevertheless allows one to exclude 1 min periods where wind might influence sound propagation. At the same time, selecting measurements at low wind speeds only also prevents microphone induced wind noise. A maximum value of the wind speed equal to $0.5 \mathrm{~m} / \mathrm{s}$ was used. To exclude measurements made during precipitation, only those measurements made when relative humidity was lower than $80 \%$ were retained. As a result, the comparison between prediction and measurement will be limited to dry, windless conditions.

Temperature profiles were obtained by means of eight ventilated temperature sensors attached to the posts of a cable way (the "Kellerjoch Bahn"). The heights of the sensors ranged from $540 \mathrm{~m}$ (valley floor) to $1341 \mathrm{~m}$. The Kellerjoch Bahn is located North-West from cross section 1 and cross section 2, at 4.5 and at $6.5 \mathrm{~km}$, respectively. Every $15 \mathrm{~min}$, the temperature at all heights was logged simultaneously. Thus, air temperature is known along a single line on a slope. In an ideal situation, temperature should be measured at a fixed location (e.g., in the center of the valley) at different heights but this was not practically achievable.

\section{B. Noise measurements and processing}

In each cross section, simultaneous measurements were performed with Svantek 1/2 in. SV22 condenser microphones. Overall equivalent A-weighted sound pressure levels are stored every second $\left(L_{\text {Aeq, } 1 \mathrm{~s}}\right)$.

Besides the main road noise that is of interest for this study, noise from train passages and local events like the passing of a car on a nearby, small road are also recorded by the microphones. To eliminate these disturbances, the raw $L_{\text {Aeq,1 s }}$ measurements are pre-processed based on the hypothesis that the main road noise under study constitutes the constant part of the sound level. The following rule was used: The sound pressure level at a given second is consid- ered to be an event if it is higher than the median noise level in a time window of $5 \mathrm{~min}$, centered on the second under consideration, plus $5 \mathrm{dBA}$. After events were removed in this way, sound pressure levels were summed to 1 min equivalent sound pressure levels. Only if at least $40 \mathrm{~s}$ of measurements remain after event canceling, the 1 min equivalent sound pressure level is kept for further analyses.

\section{Combined dataset}

The measurement campaign led to a dataset of combined noise and meteorological data. A clustering of these data was done based on (similar) range-independent temperature profiles, in order to limit the number of calculations. The minimum number of data points in each cluster was set to 50 to allow one to draw statistically stable conclusions. The parameters used for clustering were the gradients in air temperature between successive sensors, up to a height of $767 \mathrm{~m}$. Gradients at larger heights did not influence sound propagation over the distances and height differences considered. The (absolute) value of temperature may differ within a cluster. This is acceptable since the gradient in temperature is responsible for refraction of sound.

The preprocessing of the dataset to retain windless periods without precipitation, together with the demand that at least 50 measurements are characterized by similar air temperature profiles, resulted in a drastic decrease of available combined noise and meteo data. The 4270 measurements (available after removing events) in cross section 1 resulted in 53 usable combined data records. For cross section 2, 21,601 measurements gave 274 combined data records, split up into three temperature profiles clusters.

In Table II, the number of (non-successive) $1 \mathrm{~min}$ combined data records are shown for the different clusters considered. During all of these selected periods, the ground was snow-covered.

The data for cross section 1 was measured on a single day, between 16.00 and $19.00 \mathrm{~h}$. For cluster 2, data come from different days and were recorded during day hours. The data in cluster 3 come again from a single day, around noon. Cluster 4 contains mainly observations during night hours. 


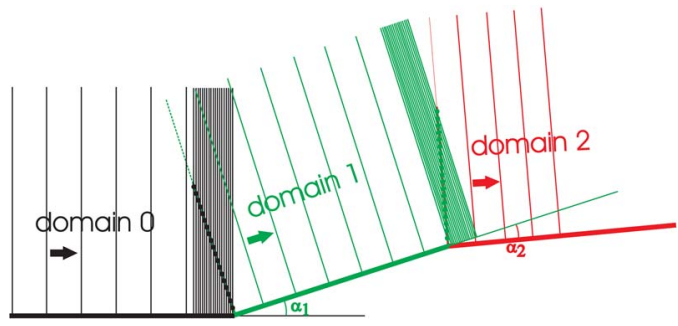

FIG. 2. (Color online) Schematic representation of sound propagation with the GFrPE method in the case of concave and convex ground surface transitions. Three successive flat domains are shown. The vertical lines represent the positions where a column of pressures is calculated. In the transition zones, a large number of propagation steps are needed; $\alpha_{i}$ represent the difference in slope angle between successive domains.

\section{NUMERICAL PREDICTIONS}

\section{A. GFrPE method}

A two-dimensional Green's Function Parabolic Equation $(\mathrm{GFPE})^{5,6}$ method with a rotated reference frame (abbreviated as GFrPE) is used for the numerical predictions. In this model, the undulating terrain is approximated by a succession of flat domains with different slopes. In each of them, ordinary GFPE calculations are performed. As shown in Fig. 2 , the sound field calculation in each domain starts from an array of pressure values, orthogonal to the local slope. The starting field for domain $n+1$ is constructed based solely on calculations in domain $n$, which is in line with the progressive character of the PE method. When there is a change in the slope angle between successive domains, a number of reduced propagation steps are needed in domain $n$ near the transition to the next domain, to allow one to obtain the pressures at the correct heights for constructing the starting field for domain $n+1$.

This methodology is illustrated in Fig. 2, for both a concave transition (from domain 0 to 1 ) and a convex transition (from domain 1 to 2). In the case of a convex ground surface, PE calculations are necessary along the (virtual) continuation of the ground in domain $n$ for calculating the pressures of the starting field of domain $n+1$.

This modification to the GFPE method was proposed in Refs. 7 and 8. The GFrPE method has the same benefits as GFPE:

- The computational cost of GFPE is based mainly on the efficiency of the fast Fourier transform (FFT) algorithm. Very fast FFT algorithms are available.

- GFPE allows one to use large step sizes in horizontal direction, that are limited by the inhomogeneity of the atmosphere rather than by the sound wavelength $(\lambda)$. The maximum acceptable range step varies roughly between $5 \lambda$ and $50 \lambda$ in a refracting atmosphere. ${ }^{6}$ Since we are aiming at distances up to a few kilometers from the source and for typical traffic noise (including relatively high frequencies), the use of large step sizes is an important advantage. Section IV B discusses how this advantage could be jeopardized by the use of GFrPE.

- Refraction of sound by arbitrary sound speed profiles can be modeled: The sound speed profiles may contain upward and downward refracting parts.

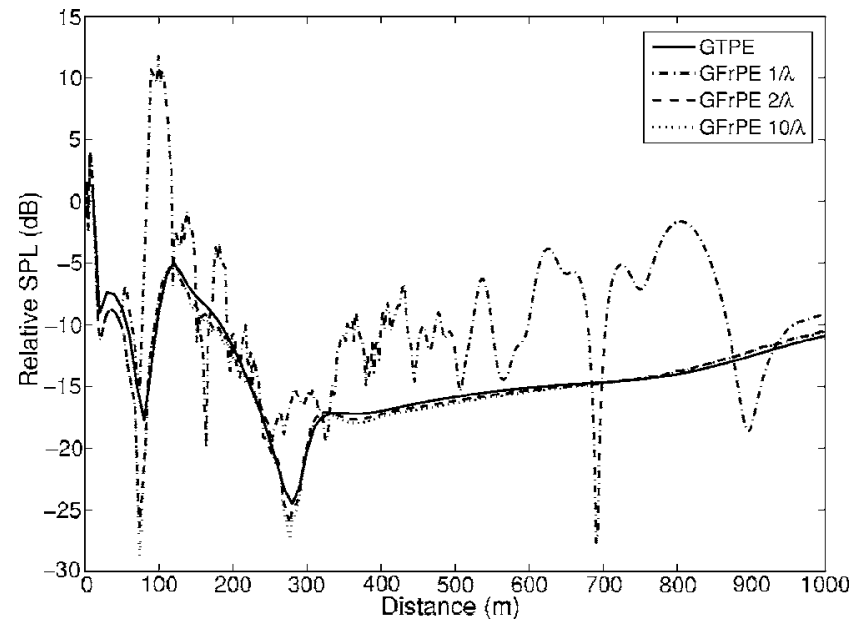

FIG. 3. Effect of the number of calculations per wavelength $(n / \lambda)$ on the starting fields for GFrPE calculations. See the caption of Fig. 11 for more details on the calculation parameters. The GTPE calculation is used as a reference solution. When two points per wavelength or more are calculated, accuracy is not improved anymore.

- Locally reacting, range-dependent impedance planes can be used to model reflection from the ground.

- Diffraction near hard, infinitely thin screens can be modeled with the Kirchoff approach. ${ }^{9}$

The method was validated for typical road embankment configurations by comparing it with results obtained using the boundary element method. ${ }^{8}$ In the Appendix, the GFrPE code is validated for the case of the smooth hill presented in Ref. 10.

\section{B. Improving computational efficiency of GFrPE}

The construction of the starting field for domain $n+1$ from field calculations in domain $n$ is computationally very costly. As shown in Fig. 2, a large number of very small propagation steps are needed in the transition zone. A typical starting field would be described by about ten values per wavelength in the vertical direction. In practice, it is not necessary to accurately calculate the field with this resolution on the basis of the propagation in domain $n$. Instead, linear interpolation in both amplitude and phase can be used to construct the starting field from a lower number of known values. In Fig. 3, the relative sound pressure level as a function of distance for the validation case, described in the Appendix, is shown, with a decreasing number of explicitly calculated points per wavelength on the starting fields. Once 2 points per wavelength (indicated as $2 / \lambda$ ) or more are calculated, the result converges.

GFrPE calculations can also be accelerated by truncating the height of the calculated starting field below the top of the computational grid (or up to the beginning of the absorbing layer). The starting pressure field above this truncation height is obtained by linear extrapolation of the phase angle of the pressure and linear tapering to zero of the magnitude of the pressure as was proposed in Ref. 11. The maximum height still containing relevant information depends on the propagation distance to be covered by the PE model. Figure 4 shows that the relative sound pressure level converges for 


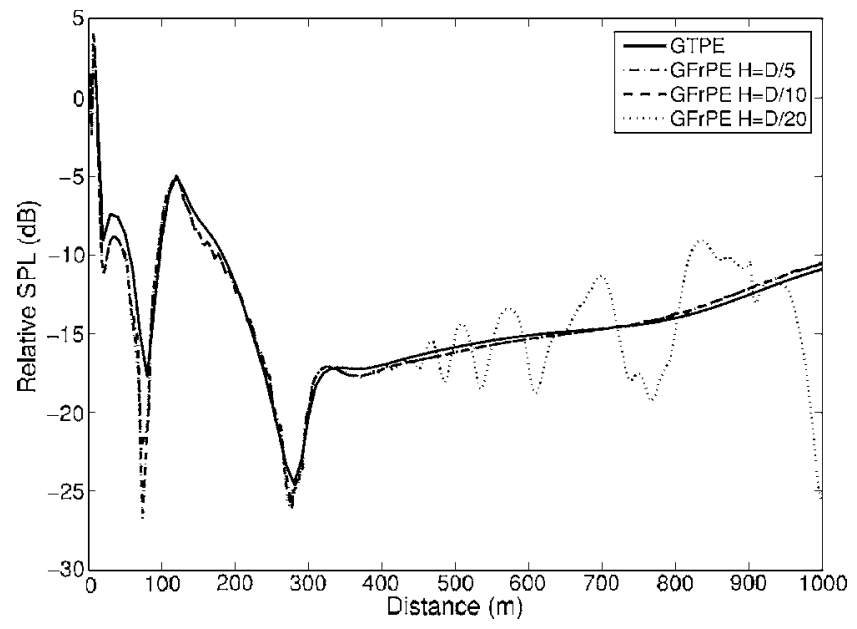

FIG. 4. Effect of the ratio between the maximum height on the starting fields taken into account $(\mathrm{H})$ and the distance between source and receiver (D). See the caption of Fig. 11 for more details on the calculation parameters. The GTPE calculation is used as a reference solution. The use of values of $\mathrm{H}$ larger than $\mathrm{D} / 10$ only increase the computational cost, while accuracy is not improved anymore.

the test case of the Appendix when the ratio between the propagation distance to be covered $(D)$ and the truncation height of the starting field $(H)$ is at least 10 . Decreasing this ratio only increases the computational cost, while there is no gain in accuracy.

\section{Comparing relative levels between predictions and measurements}

Detailed traffic counts, traffic composition, and traffic speed distribution were not available for the motorways during the measurement campaign. This lack of information can be circumvented by validating the numerical results on relative rather than absolute measurements. In each cross section, the location closest to the road was chosen as the reference measurement: microphone with ID 2 in cross section 1 , and microphone with ID 6 in cross section 2. Sound pressure levels at the distant points relative to the reference points were compared with numerical calculations.

The number of vehicles on the road will not influence the comparison of relative sound levels as proposed in the previous paragraph if it can be assumed that the flow is homogeneous over a sufficiently long stretch of the road. This does not hold for vehicle type and vehicle speed. Each combination of vehicle type and vehicle speed results in a typical source spectrum. Keeping in mind the difference in distance between reference point and the more distant points, the contributions of different frequency bands to the total sound pressure level will change. As a result, the relative sound pressure levels will vary. To account for this, the spread in sound pressure level caused by different vehicle types, driving at typical velocities, will be predicted.

\section{Calculation methodology for the valley-slope configuration}

The methodology for calculating the sound pressure levels from traffic noise at distant receivers uses a number of aspects from the HARMONOISE reference model. ${ }^{12}$
First the three-dimensional propagation problem is split up into a set of two-dimensional problems by subdividing the roads in a number of point sources along its axes.

In a second step, the terrain profile along the line between each point source location and the receiver is extracted from the digital terrain map. In GFrPE, the terrain is approximated as a succession of flat segments, each with a different slope.

The points of intersection between a source-receiver line and obstacles (buildings, highway screens and railway screens) are determined. The GFrPE method is able to handle diffraction over thin, hard screens using the Kirchoff approach: The acoustic field is set to zero on the barrier, and then propagated in forward direction. For simplicity, a building is approximated by such a thin, hard screen located in the center of the building. In this two-dimensional approach, only diffraction over the top of the obstacles is accounted for. It is also assumed that the obstacles are rotated in such a way that their main axis becomes perpendicular to the sourcereceiver line. These same assumptions were made in the HARMONOISE reference model ${ }^{12}$ (except when a threedimensional ray model is used).

In a third step, the transmission loss from each point source to the receiver is calculated using GFrPE. Temperature profiles are available on a single line along a slope. For simplicity, horizontal temperature stratification throughout the valley is assumed: Only the elevation determines the air temperature. The validity of this approach could be questioned, but it remains the best possible approach with the available meteorological data.

The highest temperature sensor in our case was located at a height of $1341 \mathrm{~m}$ (or $801 \mathrm{~m}$ above the valley floor). This large span in heights of temperature observations is more than sufficient to accurately model refraction of sound, keeping in mind the distances between sources and receivers. The temperature and sound speed at each position in the vertical $\mathrm{PE}$ grid is obtained by linear interpolation between measured data.

Atmospheric attenuation is not included in the GFrPE model, and is therefore added afterwards, using the product $\alpha r$, where $\alpha$ is the absorption coefficient which is calculated following ISO $9613-1^{13}$ and $r$ is the distance traveled by the direct sound ray between source and receiver. All combinations of air temperature, relative humidity and air pressure that are present in each temperature profile cluster are considered.

In the case of an upward refracting atmosphere, turbulent scattering into the acoustic shadow zone that is formed becomes important. Neglecting this effect often results in unrealistically large attenuations. A standard approach to account for turbulent scattering consists in calculating sound propagation through a number of turbulent realizations of the atmosphere (see, e.g., Ref. 14). The ensemble average of all these realizations follows statistical laws. To have statistically stable results however, at least 50 realizations of the turbulent atmosphere need to be taken into account. As a result, computing times increase dramatically.

Based on experiments, it was found that the sound pressure level relative to free field propagation stays more or less 
constant in the acoustical shadow zone formed by an upward refracting atmosphere. ${ }^{15,16}$ This constant value depends on the geometry of the problem and on the strength of the turbulence. A value of $-20 \mathrm{~dB}$ is common in acoustical literature. ${ }^{10}$ Truncating the sound pressure level relative to free field comes at no additional computational cost and is therefore preferred for the large scale problem considered in our work. It was observed that this approach led to acceptable results in situations where turbulent scattering into shadow zones becomes important (see further).

In a fourth step, the sound pressure level at the receivers is calculated by choosing an appropriate traffic source spectrum.

Finally, the contributions from all two-dimensional cross sections are added incoherently to find the total sound pressure level at the receiver.

This approach assumes that in each point source, the same type of vehicle is present driving at the same speed. When multiple vehicle types and vehicle speeds are considered, step four (and five) are repeated with an appropriate source spectrum.

\section{E. Model parameters}

The numerical parameters were chosen as a compromise between numerical accuracy and computational efficiency.

\section{Frequency range}

One-third octave bands ranging from 50 to $2500 \mathrm{~Hz}$ were considered, covering sufficiently the frequency spectrum of road traffic. Propagation calculations were performed for a single frequency per one-third octave band. The calculations were repeated in the first cross section using three frequencies per one-third octave band for validation. This resulted in differences in total A-weighted sound pressure levels that were less than $0.3 \mathrm{dBA}$, while the computational cost was three times higher. Thus it was decided to use a single frequency per one-third octave band in all further predictions.

\section{Source and receiver heights}

A source height above the road surface of $0.5 \mathrm{~m}$ was chosen to represent an average over all possible physical noise sources at different speeds for different types of vehicles. The HARMONOISE reference model ${ }^{12}$ suggests using different source heights for each source mechanism (rolling noise, engine noise) and each class of vehicles (cars, light trucks and heavy trucks). Since each source height would result in a new propagation calculation, this suggestion was not followed here. The receiver height at all locations was $2 \mathrm{~m}$, except for the microphone with ID 6, which was placed $2 \mathrm{~m}$ above the roof of a small building.

\section{Discretization of the roads}

The stretch of road considered to contribute to the overall noise level was limited by the furthest points being at a distance from the receiver equal to three times the orthogonal distance between road and receiver. In the case of direct sound and in a still and homogeneous atmosphere, a source at this distance has a contribution to the overall sound level that is nearly $10 \mathrm{~dB}$ below the largest contribution. The discretization distance between successive source points along the road axis was first chosen to be $200 \mathrm{~m}$. The number of source points that were used for the different microphone positions ranges from 9 to 35 with such a discretization along the road and using such a marking off. This rough sampling of point sources along the road revealed the zone with the most important contributions, which was in most cases centered around the source point with the shortest distance to the receiver. The stretch of road between the first and the last source point resulting in a sound pressure level of $10 \mathrm{dBA}$ below the most contributing source was subject to further refinement. Additional source points were placed every $100 \mathrm{~m}$. If the total A-weighted sound pressure level obtained using the refined road discretization deviated less than $1 \mathrm{dBA}$ from that obtained using the rougher discretization, convergence was assumed. If not, the refinement procedure was repeated. In most situations a single refinement proved sufficient.

\section{Discretization of sound paths}

Along each sound path between the source and receiver, the terrain is approximated by flat domains with a length of $100 \mathrm{~m}$. This approximation is acceptable because the relief is reasonably smooth up to the microphone positions. Taking smaller segments largely increases the computational cost mainly because of the smaller spatial step and corresponding larger number of calculations needed in each transition zone.

\section{Obstacles}

The railway screens in the area under consideration have a height of $3 \mathrm{~m}$. The height of the highway noise barriers ranges from 3 to $4.5 \mathrm{~m}$. The height of individual buildings and houses is not known. An average height of $5 \mathrm{~m}$ is chosen. All obstacles are considered to be rigid.

\section{Ground modeling}

A range-dependent ground impedance is used. The ground directly under the source points is assumed rigid. The river, which is close to the roads, is modeled as a rigid surface as well. All remaining grounds in the source-receiver lines are assumed to be covered with snow. The soil in the buildup areas is not considered separately.

Sound propagation over snow-covered ground has been investigated in detail in acoustical literature. ${ }^{17,18}$ Information on, e.g., the thickness of the snow layer or its state (old snow, fresh snow, the degree of compaction, etc.) was not available during the measurement campaign. Therefore, the generalpurpose one-parameter ground impedance model of Delany and Bazley ${ }^{19}$ was used, with a measured flow resistivity for snow equal to $30 \mathrm{kPa} \mathrm{s} / \mathrm{m}^{2}$ (Ref. 12).

\section{Traffic source spectrum}

The NORD 2000 traffic source spectra ${ }^{20}$ are used. The range of differences in total A-weighted sound pressure level between the distant receivers and the reference receiver are calculated for passenger cars driving at 70, 90, 110, and 
$130 \mathrm{~km} / \mathrm{h}$, for medium-heavy vehicles driving at 70,90, and $110 \mathrm{~km} / \mathrm{h}$, and for heavy vehicles driving at 50, 70, and $90 \mathrm{~km} / \mathrm{h}$.

\section{GFrPE parameters}

A standard Gaussian starting function ${ }^{5,6}$ is used to initiate the GFrPE calculations. The absorbing layer as described in the Appendix is applied. The maximum horizontal propagation step is $10 \lambda$, and is used as much as possible. Variable horizontal propagation steps are used in order to exactly account for the locations of screens, houses, receivers and impedance changes.

The optimization of characteristic parameters for the GFrPE method was discussed in Sec. IV B. To construct a new starting field for the next domain, two calculations per wavelength are performed in vertical direction followed by linear interpolation to obtain a vertical discretization of $0.1 \lambda$. The maximum height of the starting field that is calculated was one tenth of the distance between source and receiver. The starting field above this maximum height is found by extrapolation, as described in Sec. IV B.

A change in slope inclination between successive domains of less than $1^{\circ}$ is ignored. The starting field for domain $n+1$ is in that case the vertical array of pressures at the border of domains $n$ and $n+1$.

\section{Parallel computations}

The proposed calculation methodology is computationally costly. This holds especially for the highest frequencies considered, for the oblique (and thus long) sound paths and for the sound paths with a large number of differences in slope inclination between successive domains. In the proposed methodology, calculations are easy to parallelize. Either different sound paths were calculated on different CPU's, or different frequencies were calculated on different CPU's.

\section{COMPARISON BETWEEN MEASUREMENTS AND PREDICTIONS}

An overall comparison between measurements and predictions is shown in Figs. 5(a)-5(d). The measurements are presented by means of so-called boxplots. The (middle) horizontal line in the box indicates the median of the data. The box is closed by the first and third quartile. The whiskers extend to 1.5 times the interquartile distance above the maximum value inside the box, and to 1.5 times the interquartile distance below the minimum value inside the box. Data points that fall outside the whiskers are considered to be outliers, and are indicated with the plus signs.

Three sets of predicted relative noise levels are included in the plots, indicated by different symbols. The first set is the best available prediction. To gain understanding in the significance of the terrain elevation and temperature effects, additional calculations were performed. First, a homogeneous atmosphere was assumed in the presence of the actual relief. Second, a flat terrain is assumed in a homogeneous atmosphere. In both calculations all other numerical and geometrical parameters (locations of houses and screens, ground impedance, etc.) remain unchanged.

As discussed in Sec. III C, the combined noise-meteo measurements were clustered based on the temperature profiles. This still leaves an important variance in the relative measurements. The reasons for this variance are summarized below:

- Traffic composition and vehicle speed may be different during the non-successive minutes considered, resulting in different source spectra.

- In each cluster, there is a variation in the combination of air temperature, relative humidity and atmospheric pressure, which is responsible for the magnitude of the atmospheric absorption. An additional clustering on this data would lead to too few cases per cluster.

- An average, clustered temperature profile is chosen for the calculations [see full lines in the temperature profile plots in Figs. 5(a)-5(d)]. Within each cluster, temperature profiles might be slightly different [see dashed lines in the temperature profile plots in Figs. 5(a)-5(d)].

The first two causes of variance in the relative sound pressure levels are accounted for (see Sec. IV C and IV D) and result in the spread in calculation results that can be observed in Figs. 5(a)-5(d). The last one is not included for reasons of computational cost.

The agreement between measurements and numerical predictions is good. The average of the calculations lies close to the median of the measurements. Differences range up to $2-3 \mathrm{dBA}$. The spread in the calculations is in most situations very similar to the measured one. This gives confidence in the followed approach.

A comparison between the flat terrain calculations and the calculations using the actual relief, both in a homogeneous atmosphere, indicates that the presence of the sloping terrain is responsible for an increase in sound pressure level for the distant observation points of at least $5 \mathrm{dBA}$ and at most $30 \mathrm{dBA}$. Comparing the calculations in the case of a homogeneous and an inhomogeneous atmosphere, both using the actual relief, reveals that temperature gradients in this Alpine valley result in a change of sound pressure level ranging from $-3 \mathrm{dBA}$ to $+10 \mathrm{dBA}$ between the distant and reference receiver.

The temperature profiles in the dataset are complex: They contain upward and downward refracting parts, depending on the height. Temperature profiles 1 (see Fig. 5(a)) and 4 (see Fig. 5(d)) are mainly upward refracting. Very close to the ground, a thin temperature inversion layer is observed. This holds also for temperature profile 2 (see Fig. $5(\mathrm{~b})$ ); in addition, starting from about $100 \mathrm{~m}$ above the valley floor, a temperature inversion layer is observed. Temperature profile 3 (see Fig. 5(c)) is characterized by a well-mixed, unstable layer starting from the ground surface, capped by a strong inversion layer, starting at the same height as in temperature profile 2 .

In the first cross section, the relief increases the sound pressure levels at the distant points 3 and 5, relative to the reference point 2. This is shown in Fig. 5(a). When the tem- 

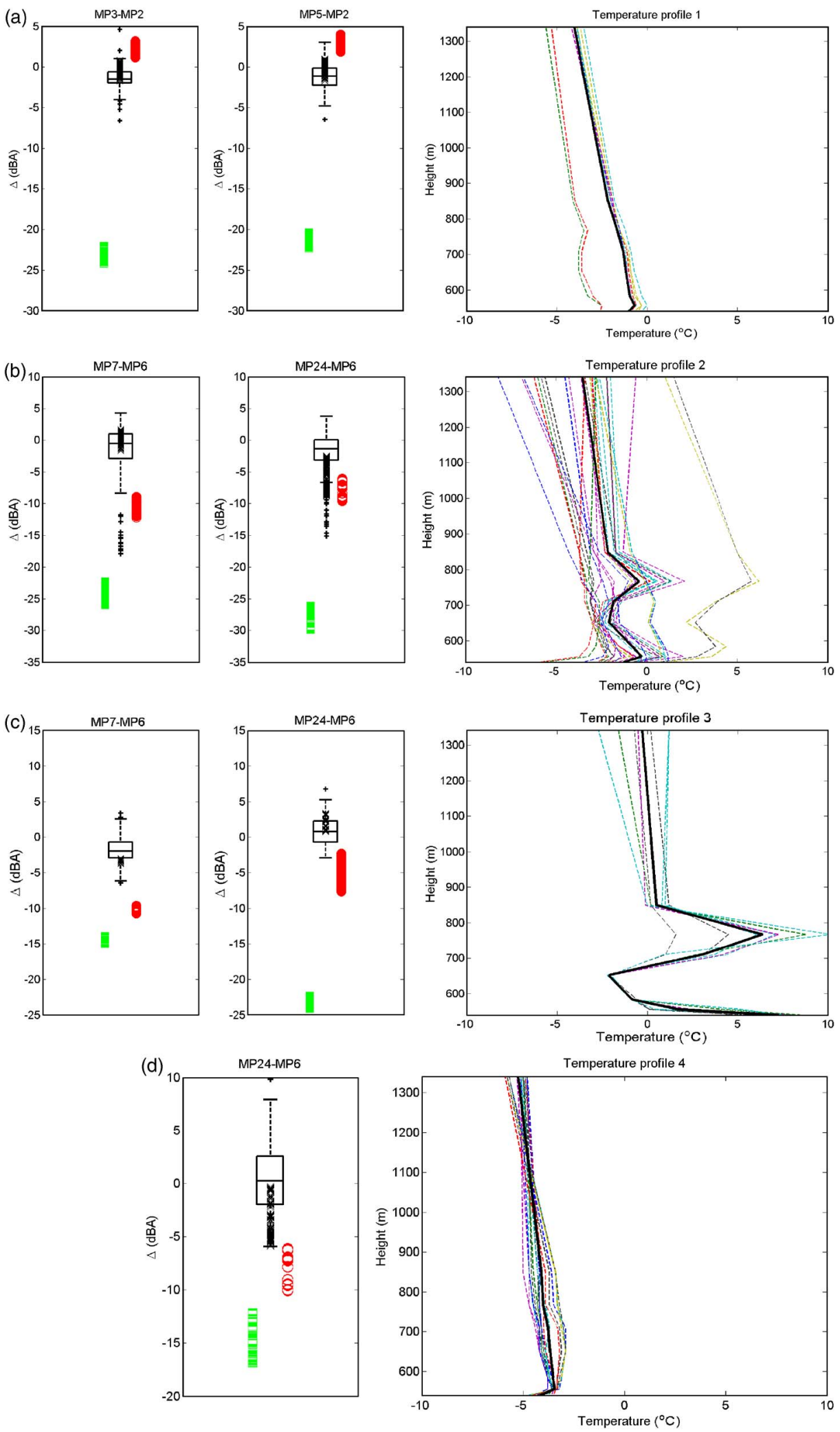

FIG. 5. (Color online) The figures on the left show a comparison between measurements and calculations. The measurements are represented by means of boxplots. +- signs indicate outliers of the measurements. The series of $\mathrm{x}-$ signs indicate the calculations accounting for the actual relief and the actual temperature profile (that can be compared to the measurements). The series of $\bigcirc-$ signs indicate the calculations with the actual relief in a homogeneous atmosphere, the series of $\square$-signs indicate the calculations with a flat terrain in a homogeneous atmosphere. The figures on the right show the temperature profiles forming the clusters (dashed lines), together with the average temperature profiles that were used for the calculations (thick full lines). In part (a), cross section 1 is considered. The sound pressure levels at measuring points (MP) 3 and 5 are shown, relative to the sound pressure level at MP 2 (closest to the road). In parts (b), (c), and (d), cross section 2 is considered. In parts (b) and (c), the sound pressure levels at MP 7 and MP 24 are shown relative to MP 6, for two temperature profile clusters. In part (d), the sound pressure level at MP 24 relative to MP 6 is shown, for temperature profile cluster 4 . perature profile is included, a shift in the other direction is observed. The sound pressure level at microphone 2 slightly increases because of the thin temperature inversion layer very close to the ground. Microphones 3 and 5 receive less sound, because of the upward refracting atmosphere. As a result, the difference in sound pressure level, relative to the homogeneous atmosphere (with relief), decreases, and a relative sound pressure level near $0 \mathrm{dBA}$ is found.
In cross section 2 (see Figs. 5(b)-5(d)), the effects of both the elevation of the terrain and the inhomogeneous atmosphere tend to decrease the difference in sound pressure level between the reference point and the more distant points. Qualitative analysis of what is happening is difficult because of the complex interaction of the mechanisms involved. The terrain profiles corresponding to the different source-receiver lines for microphone 24 are complex, and are 


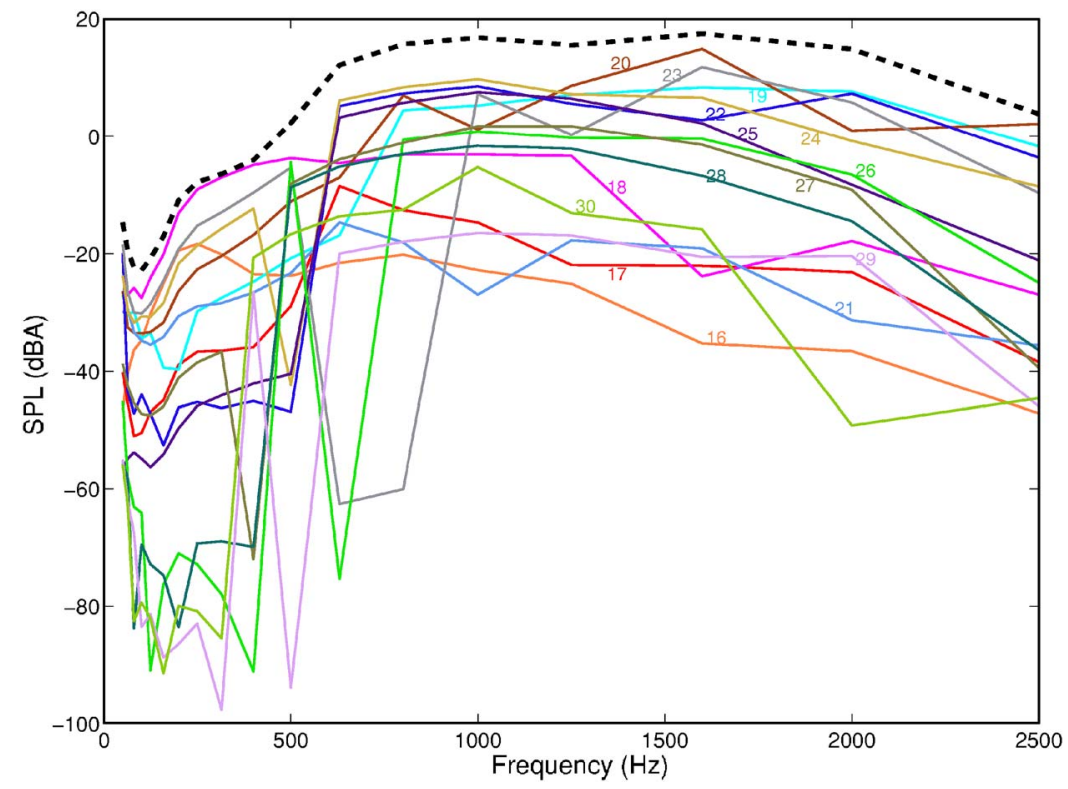

FIG. 6. (Color online) Calculated frequency spectrum at receiver 3 for the 15 most contributing sound paths. A passenger car at $110 \mathrm{~km} / \mathrm{h}$ is assumed in each source point. The temperature is $0{ }^{\circ} \mathrm{C}$, the relative humidity is $65 \%$. Temperature profile 1 is used. The total A-weighted spectrum is indicated by the thick, dashed line. The magnitudes of the sound pressure levels are arbitrary. Source points after the refinement are not shown. For each source point, the frequency spectrum is clearly different.

characterized by a sudden increase in the elevation of the ground, close to the receiver. These profiles further contain successions of pronounced concave and convex parts. The strongly upward refracting part in temperature profile 3 (see Fig. 5(c)) results in an acoustic shadow zone. Limiting the attenuation in this situation is important to account for turbulent scattering (see Sec. IV D). The situation is further complicated because microphone 6 was placed on the roof of a building. This receiver was (slightly) shielded by the edge of the roof, and this intensifies differences in sound pressure level by the different states of the atmosphere. In contrast to a situation with direct sound, the contribution caused by atmospheric refraction, although small, results in an important increase in the sound pressure level. A similar conclusion could be drawn in the case of sound propagation between adjacent street canyons in an urban environment. ${ }^{21}$ The numerical model nevertheless manages to produce sufficiently accurate results.

In both cross sections, the medians of the measured relative sound pressure levels range from -3 to $3 \mathrm{dBA}$. The relief in combination with the refracting atmosphere compensate for the effect of geometric divergence of the sound wave, ground attenuation and atmospheric absorption.

Note that the calculated relative sound pressure levels also change considerably in the case of a homogeneous atmosphere, because of changes in the magnitude of and the variation in atmospheric attenuation during the periods of the different temperature profile clusters. In the case of temperature profile 4 in cross section 2 (see Fig. 5(d)), the high and constant relative humidity in that period induces almost no atmospheric absorption. The variation in the calculations in that case is caused only by differences in the modeled source spectrum. In the other situations in cross section 2 (see Figs. 5(b) and 5(c)), lower values of relative humidity also occur, leading to more atmospheric absorption. As a result, about $10 \mathrm{dBA}$ difference can be observed between the different clusters when considering the relative sound pressure levels at microphone 24 in the case of a flat and homogeneous atmosphere.
The calculations also allow one to have a closer look at, e.g., frequency spectra. In Fig. 6, the computed sound pressure levels of the 15 most contributing sound paths to the overall sound pressure level at receiver 3 are shown, as a function of frequency. Detailed geometrical information is shown in Figs. 7 and 8: the source-receiver lines plotted on the orthophotos, and the terrain profile between each source point and the receiver. The sound pressure level frequency spectrum for each sound path is clearly different. In Fig. 9, this same information is compacted to total A-weighted sound pressure levels per sound path. The sum of all sound paths (which is the data that are used for the comparison with measurements) is indicated with the horizontal line. The source point closest to the receiver is number 17. Its contribution to the total sound pressure level at the receiver is smaller than, e.g., for sound path 20 . On the other hand, an important contribution could have been expected from point 21. As is clear from Fig. 9, this source point is not contrib-

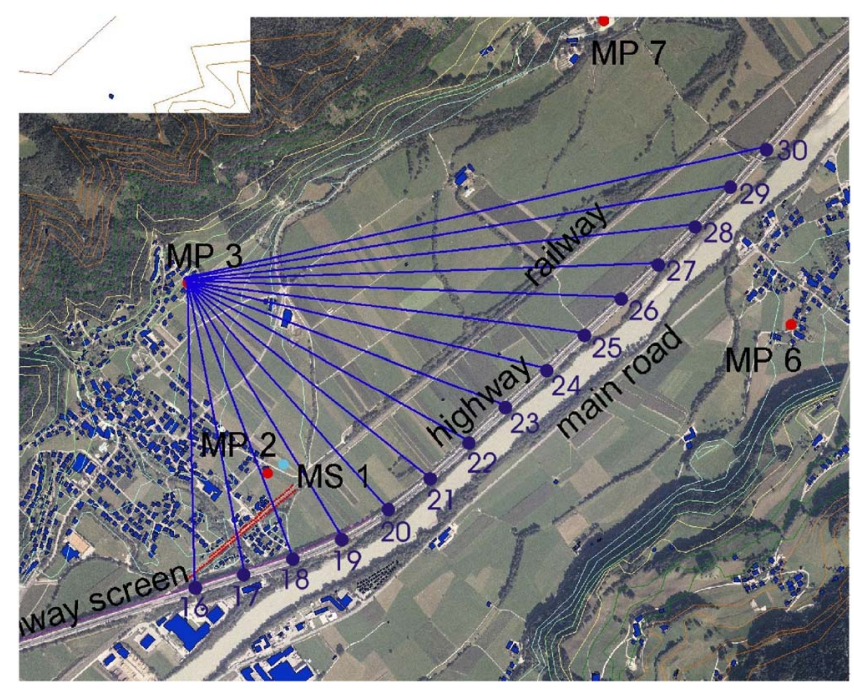

FIG. 7. (Color online) Orthophoto of the 15 source points under consideration for microphone position 3 . The source points after refinement are not shown. Height contours are shown on the map. 

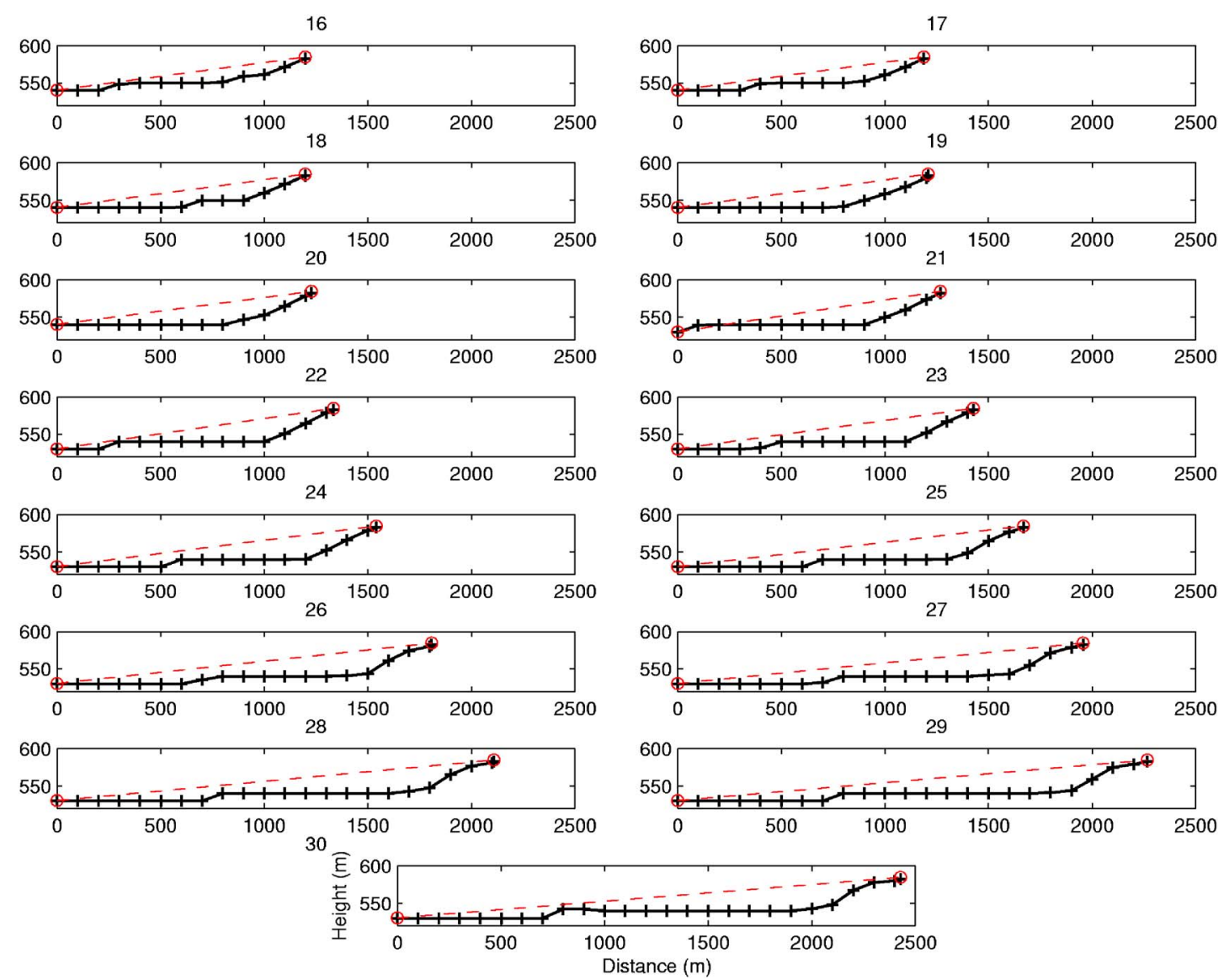

FIG. 8. (Color online) Terrain profiles for the 15 source points under consideration for microphone position 3 . The distance towards the source is shown on the horizontal axis, the height is shown on the vertical axis. The source and the receiver (open circles) are connected with a straight, dashed line. The vertical axis is not true to scale. Source points after refinement are not shown.

uting to the sound field at the receiver; the difference in sound pressure level relative to source point 20 is more than $25 \mathrm{dBA}$. The relief is responsible for this. Starting from source point 21 , the road becomes somewhat depressed. As a result, sound is shielded effectively at source point 21: There is no direct view between source and receiver. This analysis

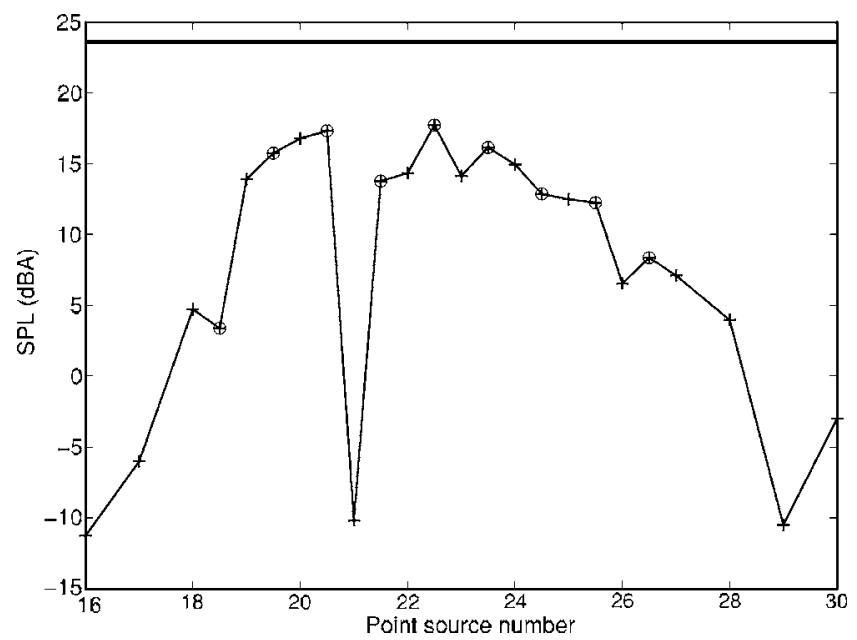

FIG. 9. Total A-weighted sound pressure level resulting from different sound paths, at microphone position 3. A description of the parameters involved is found in the caption of Fig. 6. The source points after refinement are shown with the open circles. The sum of all profiles is indicated with the horizontal line. The magnitudes of the sound pressure levels are arbitrary. of the source points contributing to microphone position 3 clearly shows that the degree of detail included in our calculations is necessary.

\section{CONCLUSIONS}

In this paper, a comparison is made between measured and calculated sound pressure levels in a valley-slope configuration, during windless periods without precipitation. A rotated Green's Function Parabolic Equation (GFrPE) method was used for the numerical calculations. Typical parameters related to GFrPE like the number of actual calculations needed near the transition of successive domains, and the maximum height to be considered on the starting fields, were optimized to increase computational efficiency.

A methodology is presented to calculate sound pressure levels from road traffic. This calculation methodology is related to the HARMONOISE reference model. The road traffic noise source is discretized by a number of point sources, and two-dimensional calculations are performed for each source-receiver vertical plane. The detailed sound propagation calculations include the undulation of the terrain, the presence of obstacles (like noise screens and houses), (ground) impedance discontinuities, and refraction by arbitrary sound speed profiles.

The agreement between numerical calculations and measurements is good; differences are smaller than $3 \mathrm{dBA}$. The 


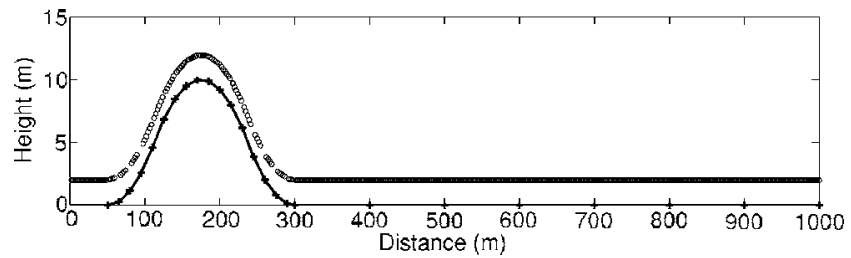

FIG. 10. Terrain profile used for the validation of the GFrPE model. The vertical axis is not true to scale. The open circles indicate the receiver positions

spread of the sound pressure levels is similar for both the measurements and the calculations. Additional calculations allow one to separate effects from the relief and the refractive state of the atmosphere. The elevation of the terrain is responsible for an increase in the sound pressure level at distant points up to $30 \mathrm{dBA}$. The temperature profiles observed in this mountainous area are complex and contain upward and downward refracting parts. Their influence ranges from $-3 \mathrm{dBA}$ to $+10 \mathrm{dBA}$. The sound pressure levels at the distant points, relative to the reference points, are in most cases near $0 \mathrm{dBA}$. The relief in combination with the refracting atmosphere compensates the expected decrease in sound pressure level caused by geometric spreading of sound, ground attenuation and atmospheric absorption.

The detailed validation of the GFrPE model for valleys in mountainous areas shows both that the proposed model is accurate enough to predict sound levels up to distances of $1000 \mathrm{~m}$ and that further simplification of full wave calculations is not possible. Although computational requirements are strongly reduced by careful tuning of the numerical parameters, the GFrPE method remains too computationally demanding to be used in noise mapping. The method is nevertheless very well suited as a reference model to which an engineering approach-to be used in the noise mapping process - can be validated, also in this particular valley-slope context.

\section{ACKNOWLEDGMENTS}

The authors would like to thank Matthias Walch for performing the field measurements, and Johannes Rüdisser (from Medical University of Innsbruck, Section of Social Medicine) and Luc Dekoninck (from Ghent University, Department of Information Technology) for help with geographical data issues. We acknowledge the administrative support of the Tyrolean government, the ASFINAG and the Brenner Eisenbahngesellschaft (BEG) for providing data. Furthermore, we thank for the financial support received from the Section of Social Medicine, Medical University of Innsbruck.

\section{APPENDIX: VALIDATION OF THE GFrPE METHOD}

The GFrPE code is validated for the case of the smooth hill presented in Ref. 10. The height of the top is $10 \mathrm{~m}$, and the hill is stretched over a distance of $200 \mathrm{~m}$ (see Fig. 10). A reference solution with the General Terrain Parabolic Equation method (GTPE) ${ }^{22}$ in this configuration is found in Ref. 10. The effect of this hill on sound propagation is significant, notwithstanding the fact that the height of the top is only

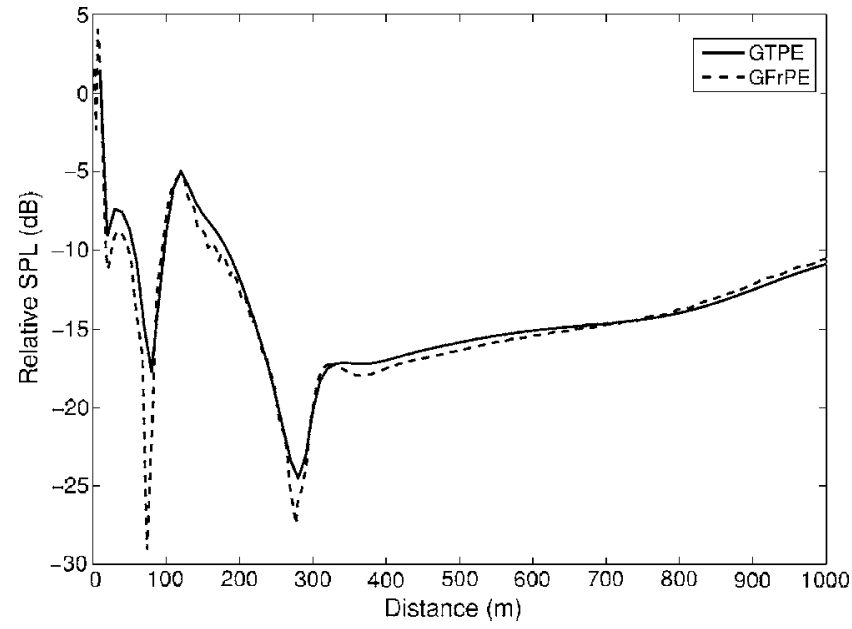

FIG. 11. Comparison between GTPE and GFrPE results in the case of the configuration shown in Fig. 10. The sound pressure level, relative to free field sound propagation, is shown with distance, for a sound frequency of $300 \mathrm{~Hz}$. The one-parameter Delany and Bazley ground impedance model is used with a flow resistivity of $200 \mathrm{kPa} \mathrm{s} / \mathrm{m}^{2}$, in a downward refracting atmosphere.

$10 \mathrm{~m}$ and that the slope angles are limited. The source is situated $2 \mathrm{~m}$ above the ground; a set of receivers is placed $2 \mathrm{~m}$ above the ground as well, up to a distance of $1000 \mathrm{~m}$ from the source. The sound frequency is $300 \mathrm{~Hz}$. The oneparameter Delany and Bazley ground impedance model ${ }^{19}$ is used with a flow resistivity of $200 \mathrm{kPa} \mathrm{s} / \mathrm{m}^{2}$. A logarithmic, sound speed profile $340+\ln (z / 0.1+1) \mathrm{m} / \mathrm{s}$ is used, where $z$ is the height above the ground in meters.

The following parameters are used during the GFrPE calculations. A standard Gaussian starter ${ }^{5,6}$ is applied. In vertical direction, ten points per wavelength $(\lambda)$ are used. The horizontal propagation step is $2 \lambda$. Every $15 \mathrm{~m}$, a new flat domain is used to discretize the hill (see Fig. 10). The thickness of the absorbing layer on top of the computational domain is $150 \lambda$. Inside this layer, an imaginary term is added to the wave number equal to $i A_{t}\left(z-z_{t}\right)^{2} /\left(z_{M}-z_{t}\right)^{2}$, where $z$ is the height, $z_{t}$ is the height where the absorbing layer starts, and $z_{M}$ is the top of the computational domain. The optimum choice of the constant coefficient $A_{t}$ depends on frequency and is chosen to be $0.5^{10}$

In Fig. 11, a comparison between GFrPE and GTPE is shown. The sound pressure level is expressed relative to free field sound propagation. The agreement between both models is very good. The terrain in the GFrPE calculation is not completely smooth because of the subdivision in flat segments. This causes some small, local distortions of the sound field, especially in the shadow zone of the hill. GFrPE results in a somewhat stronger destructive interference as well.

${ }^{1} \mathrm{~K}$. Heutschi, "On the sound propagation in Alpine valleys," Proceedings of Euronoise 2006, Tampere, Finland (2006).

${ }^{2} \mathrm{C}$. Whiteman, "Breakup of temperature inversions in deep mountain valleys. Part I. Observations," J. Appl. Meteorol. 21, 270-289 (1982).

${ }^{3}$ A. Colette, F. Chow, and R. Street, "A numerical study of inversion-layer breakup and the effects of topographic shading in idealized valleys," J. Appl. Meteorol. 42, 1255-1272 (2003).

${ }^{4}$ D. Heimann and G. Gross, "Coupled simulation of meteorological parameters and sound level in a narrow valley," Appl. Acoust. 56, 73-100 (1999). 
${ }^{5}$ K. Gilbert and X. Di, “A fast Green's function method for one-way sound propagation in the atmosphere," J. Acoust. Soc. Am. 94, 2343-2352 (1993).

${ }^{6}$ E. Salomons, "Improved Green's function parabolic equation method for atmospheric sound propagation," J. Acoust. Soc. Am. 104, 100-111 (1998).

${ }^{7}$ P. Blanc-Benon and D. Juve, "Outdoor sound propagation in complex environments: Recent developments in the PE method," Proceedings of Forum Acusticum 2002, Sevilla, Spain (2002).

${ }^{8}$ F. Aballéa, Propagation acoustique en milieu extérieur: Application de l'équation parabolique rapide au couplage d'effets météorologiques et de topographies complexes ("Using the fast parabolic equation in complex topographies, including meteorological effects, for sound propagation outdoors"), Ph.D. dissertation, Université de Maine, France, 2004).

${ }^{9}$ E. Salomons, "Diffraction by a screen in downwind sound propagation: A parabolic-equation approach," J. Acoust. Soc. Am. 95, 3109-3117 (1994).

${ }^{10}$ E. Salomons, Computational Atmospheric Acoustics (Kluwer, Dordrecht, The Netherlands, 2001).

${ }^{11}$ T. Van Renterghem, E. Salomons, and D. Botteldooren, "Efficient FDTD-PE model for sound propagation in situations with complex obstacles and wind profiles," Acust. Acta Acust. 91, 671-679 (2005).

${ }^{12}$ J. Defrance, E. Salomons, I. Noordhoek, D. Heimann, B. Plovsing, G. Watts, H. Jonasson, X. Zhang, E. Premat, I. Schmich, F. Aballea, M. Baulac, and F. de Roo, "Outdoor sound propagation reference model developed in the European Harmonoise project," Acust. Acta Acust. 93, 213-227 (2007)

${ }^{13}$ ISO 9613-1, "Acoustics-attenuation of sound during propagation
outdoors-Part 1," International Organisation for Standardization (Geneva, Switzerland, 1996).

${ }^{14}$ G. Goedecke, R. Wood, H. Auvermann, V. Ostashev, D. Havelock, and C. Ting, "Spectral broadening of sound scattered by advecting atmospheric turbulence," J. Acoust. Soc. Am. 109, 1923-1934 (2001).

${ }^{15} \mathrm{~F}$. Wiener and D. Keast, "Experimental study of the propagation of sound over ground," J. Acoust. Soc. Am. 31, 724-733 (1959).

${ }^{16} \mathrm{P}$. Chevret, P. Blanc-Benon, and D. Juve, "A numerical model for sound propagation through a turbulent atmosphere near the ground," J. Acoust. Soc. Am. 100, 3587-3599 (1996).

${ }^{17}$ J. Nicolas, J. Berry, and G. Daigle, "Propagation of sound above a finite layer of snow," J. Acoust. Soc. Am. 77, 67-73 (1985).

${ }^{18} \mathrm{D}$. Albert and J. Orcutt, "Acoustic pulse propagation above grassland and snow. Comparison of theoretical and experimental waveforms," J. Acoust. Soc. Am. 87, 93-100 (1990).

${ }^{19} \mathrm{M}$. Delany and E. Bazley, "Acoustic properties of fibrous absorbent materials," Appl. Acoust. 3, 105-116 (1970).

${ }^{20} \mathrm{H}$. Jonasson and S. Storeheier, Nord2000. "New nordic prediction method for road traffic noise," Technical report, SP, SINTEF, 2001. SP Rapport 2001:10.

${ }^{21}$ T. Van Renterghem, E. Salomons, and D. Botteldooren, "Parameter study of sound propagation between city canyons with coupled FDTD-PE model," Appl. Acoust. 67, 487-510 (2006).

${ }^{22} \mathrm{R}$. Sack and M. West, "A parabolic equation for sound propagation in two dimensions over any smooth terrain profile: The generalised terrain parabolic equation (GTPE)," Appl. Acoust. 45, 113-129 (1995). 